\title{
Hydrodynamic Attractors, Initial State Energy, and Particle Production in Relativistic Nuclear Collisions
}

\author{
Giuliano Giacalone* \\ Institut de Physique Théorique, Université Paris Saclay, CNRS, CEA, F-91191 Gif-sur-Yvette, France \\ Aleksas Mazeliauskas $\oplus^{\dagger}$ \\ Theoretical Physics Department, CERN, CH-1211 Genève 23, Switzerland \\ and Institut für Theoretische Physik, Universität Heidelberg, Philosophenweg 16, D-69120 Heidelberg, Germany \\ Sören Schlichting $\oplus^{\ddagger}$ \\ Fakultät für Physik, Universität Bielefeld, D-33615 Bielefeld, Germany
}

(Received 16 September 2019; revised manuscript received 11 November 2019; published 30 December 2019)

\begin{abstract}
We exploit the concept of hydrodynamic attractors to establish a macroscopic description of the early time out-of-equilibrium dynamics of high energy heavy-ion collisions. One direct consequence is a general relation between the initial state energy and the produced particle multiplicities measured in experiments. When combined with an $a b$ initio model of energy deposition, the entropy production during the preequilibrium phase naturally explains the universal centrality dependence of the measured charged particle yields in nucleus-nucleus collisions. Further, we estimate the energy density of the far-fromequilibrium initial state and discuss how our results can be used to constrain nonequilibrium properties of the quark-gluon plasma.
\end{abstract}

DOI: $10.1103 /$ PhysRevLett.123.262301

Introduction.-Understanding the equilibration of isolated quantum systems is a fundamental question that touches physical phenomena across vastly different energy scales, from microkelvin temperatures in cold atom experiments to trillion kelvin temperatures in the dense stronginteraction matter produced in ultrarelativistic nuclear collisions [1-4]. One outstanding discovery made in the field of heavy-ion collisions is that the system created about $1 \mathrm{fm} / c$ $\left(\approx 3 \times 10^{-24} \mathrm{~s}\right)$ after the impact of two relativistic nuclei can be described as a deconfined plasma of quarks and gluons (QGP) with macroscopic properties of temperature and velocity [5-8]. Such "unreasonable effectiveness of hydrodynamics" in describing the violent expansion of the QGP droplets triggered a new research area in mathematical physics devoted to the study of hydrodynamic attractors that emerge in out-of-equilibrium systems experiencing very fast memory loss of initial conditions and exhibiting a universal approach toward thermal equilibrium $[9,10]$.

In this Letter, we show that hydrodynamic attractors can be used to describe entropy production in relativistic nuclear collisions and to make robust estimates of initial-

Published by the American Physical Society under the terms of the Creative Commons Attribution 4.0 International license. Further distribution of this work must maintain attribution to the author(s) and the published article's title, journal citation, and DOI. Funded by SCOAP ${ }^{3}$. state energy before the onset of equilibration. We derive a simple formula, Eq. (6), that relates the energy density of the initial state to the measured charged particle multiplicity, $d N_{\mathrm{ch}} / d \eta$, and point out two important phenomenological consequences of this result. We show that the universal centrality dependence of $d N_{\mathrm{ch}} / d \eta$ across a wide range of collision systems can be naturally reproduced by combining the initial-state energy deposition in highenergy quantum chromodynamics (QCD) with the nonlinear entropy production during the equilibration process. Secondly, we determine the initial energy per unit spacetime rapidity, $d E_{0} / d \eta_{s}$, for different collision centralities at the Relativstic Heavy-Ion Collider (RHIC) and the Large Hadron Collider (LHC). By comparing our results to the experimentally measured $d E_{\text {final }} / d y$ in the final state, we estimate the work performed during the expansion of the system [11] and discuss how such an analysis constrains nonequilibrium and transport properties of the QGP.

Hydrodynamic attractors and Entropy production.-We describe the early time dynamics $(\tau \lesssim 1 \mathrm{fm} / c)$ of the hightemperature QCD plasma created in high-energy heavy-ion collisions by the out-of-equilibrium evolution of a boostinvariant and transversely homogeneous conformal system [12]. Energy-momentum conservation dictates that the energy density, $e=T^{\tau \tau}$, evolves according to

$$
\partial_{\tau} e=-\frac{e+P_{L}}{\tau},
$$


where $P_{L} \equiv \tau^{2} T^{\eta_{s} \eta_{s}}$ is the longitudinal pressure, and we use proper time $\tau \equiv \sqrt{t^{2}-z^{2}}$ and space-time rapidity $\eta_{s} \equiv$ $\operatorname{atanh} z / t$ coordinates. In (local) thermal equilibrium, the longitudinal pressure is directly related to the energy density via an equation of state, e.g., $P_{L}=e / 3$ for a conformal system. While for small deviations around equilibrium, the longitudinal pressure is determined by hydrodynamic constitutive relations in terms of the gradient expansion $P_{L} / e=\frac{1}{3}-\frac{16}{9}[(\eta / s) /(\tau T)]$, where $\eta / s$ is the specific shear viscosity $[9,10]$, this is generally not the case far from equilibrium, where, for instance, at early times after the collision of heavy nuclei, the system is highly anisotropic $P_{L} \ll e$ [4]. Nevertheless, new insights from microscopic equilibration studies [13-30] point to the existence of a hydrodynamic attractor [14], where the far-from-equilibrium system displays an effective constitutive equation $P_{L} / e=$ $f(\tilde{w})$ well before reaching local thermal equilibrium. Such attractor behavior has been established for a number of different microscopic theories (QCD Kinetic Theory [26-29], Boltzmann RTA [16-20], and AdS/CFT [1315]), where the time evolution on the attractor is controlled by a single scaling variable, $\tilde{w}=\tau T_{\text {eff }}(\tau) /(4 \pi \eta / s)$, where $T_{\text {eff }}(\tau)$ is an effective temperature such that $e(\tau) \equiv$ $\left(\pi^{2} / 30\right) \nu_{\text {eff }} T_{\text {eff }}^{4}(\tau)$ ( $\nu_{\text {eff }}$ is the number of effective degrees of freedom, e.g., $\nu_{\text {eff }}=16$ for ideal gluonic gas).

Based on these insights, the conservation law in Eq. (1) can be integrated, yielding a universal relation between the initial state energy density $e_{0}$ at very early times $\tilde{w}\left(\tau_{0}\right) \ll 1$ and the energy density $e\left(\tau_{\text {hydro }}\right)$ of the near thermal system at later times $\tilde{w}\left(\tau_{\text {hydro }}\right) \gg 1$

$$
e\left(\tau_{\text {hydro }}\right)=e_{0} \exp \left(-\int_{\tilde{w}_{0}}^{\tilde{w}_{\text {hydro }}} \frac{d \tilde{w}}{\tilde{w}} \frac{1+f(\tilde{w})}{\frac{3}{4}-\frac{1}{4} f(\tilde{w})}\right) .
$$

Close to equilibrium $f\left(\tilde{w}_{\text {hydro }}\right) \approx 1 / 3$, and the energy density of the longitudinally expanding plasma follows the Bjorken scaling $e(\tau)=e_{\text {hydro }}\left(\tau / \tau_{\text {hydro }}\right)^{-4 / 3}$, while the entropy density per unit rapidity, $s \tau$, remains constant [12]. Eventually, for $\tau \gtrsim R / c$, where $2 R$ denotes the transverse extent of the system, the QGP fireball starts expanding in the transverse plane and ultimately freezes out in color neutral hadrons [31]. During the transverse expansion, the QGP remains close to equilibrium and the total entropy per unit rapidity $d S / d \eta_{s}=A_{\perp}(s \tau)_{\text {hydro }}$ (where $A_{\perp}=\pi R^{2}$ ) is approximately conserved onwards from the time $\tau_{\text {hydro }}$ when the QGP can be described as an almost ideal fluid. Ultimately, on the freeze-out surface, $d S / d \eta_{s}$ becomes proportional to the produced charged hadron multiplicity, $d N_{\mathrm{ch}} / d \eta$. Therefore, the multiplicity of final-state particles emitted from the QGP is a sensitive probe of the entropy production during the preequilibrium phase.

Strikingly, the correspondence between initial-state energy density and charged hadron multiplicity can be quantified further using the theory of hydrodynamic

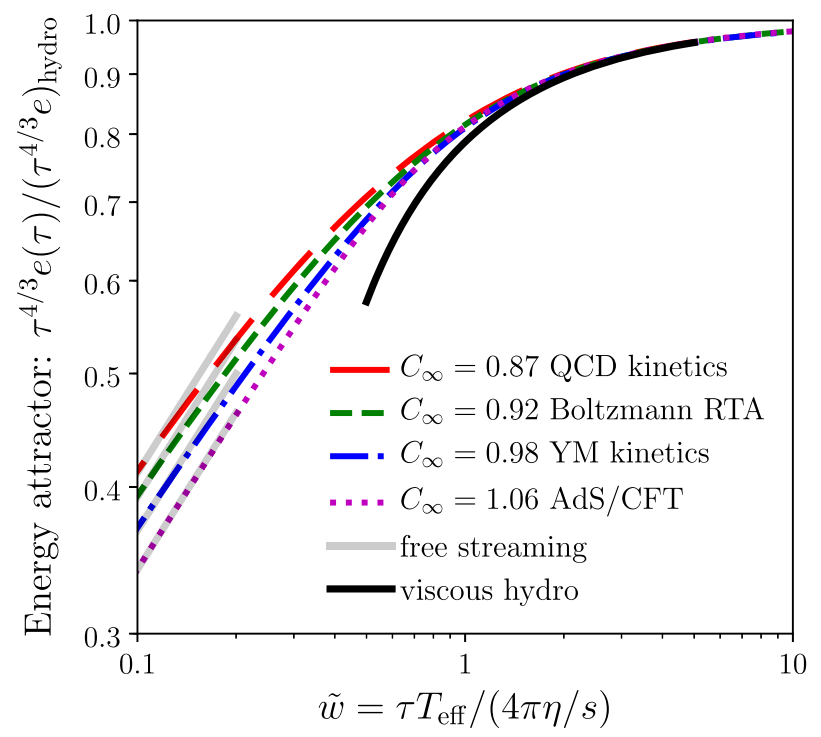

FIG. 1. Hydrodynamic attractor for preequilibrium evolution of the energy density obtained from QCD and Yang Mills (YM) kinetic theory [26-29], AdS/CFT [13-15], and Boltzmann RTA [16-20]. Solid lines show the asymptotic behavior of the attractor curves given by Eq. (4).

attractors. By factoring out the late time Bjorken scaling from Eq. (2), the evolution of the energy density during the preequilibrium phase can be characterized by an attractor curve $\mathcal{E}(\tilde{w})$

$$
\frac{e(\tau) \tau^{4 / 3}}{e_{\text {hydro }} \tau_{\text {hydro }}^{4 / 3}}=\mathcal{E}\left(\tilde{w}=\frac{T_{\text {eff }}(\tau) \tau}{4 \pi \eta / s}\right) .
$$

As can be seen from Fig. 1, the function $\mathcal{E}(\tilde{w})$ smoothly interpolates between an early free-streaming and late-stage viscous hydrodynamics $[15,26]$

$$
\begin{aligned}
& \mathcal{E}(\tilde{w} \ll 1)=C_{\infty}^{-1} \tilde{w}^{4 / 9} \quad \text { (free streaming), } \\
& \mathcal{E}(\tilde{w} \gg 1)=1-\frac{2}{3 \pi \tilde{w}} \quad \text { (viscous hydro), }
\end{aligned}
$$

where $C_{\infty}$ is a constant of order unity. Even though the evolution at intermediate times can be different for different microscopic theories, the overall similarity between different theories is remarkable. Most importantly for our purpose, all curves have the same universal characteristics, Eq. (4), at early and late times, irrespective of the underlying microscopic theory.

Based on Eq. (3), we can immediately establish a quantitative relation between the energy densities $e(\tau)$ at various stages, which, upon use of the thermodynamic relations $T s=e+p$ and $p=e / 3$ once the system is close to equilibrium, turns into an estimate of the entropy density per unit rapidity

$$
(s \tau)_{\text {hydro }}=\frac{4}{3}\left(\frac{\pi^{2}}{30} \nu_{\text {eff }}\right)^{1 / 4}\left(\lim _{\tau \rightarrow 0} \frac{e(\tau) \tau^{4 / 3}}{\mathcal{E}\left(\frac{T_{\text {eff }}(\tau) \tau}{4 \pi \eta / s}\right)}\right)^{3 / 4} .
$$


Evaluating the limit according to Eq. (4), one arrives at the central result of this Letter, namely, the relation

$$
(s \tau)_{\text {hydro }}=\frac{4}{3} C_{\infty}^{3 / 4}\left(4 \pi \frac{\eta}{s}\right)^{1 / 3}\left(\frac{\pi^{2}}{30} \nu_{\text {eff }}\right)^{1 / 3}(e \tau)_{0}^{2 / 3},
$$

from which one can directly estimate the charged particle multiplicity as discussed above

$$
\frac{d N_{\mathrm{ch}}}{d \eta} \approx \frac{1}{J} A_{\perp}(s \tau)_{\mathrm{hydro}} \frac{N_{\mathrm{ch}}}{S} .
$$

Here, $S / N_{\text {ch }} \equiv(d S / d y) /\left(d N_{\text {ch }} / d y\right) \approx 6.7-8.5$ is the entropy per charged particle at freeze-out [32] and $J \approx 1.1$ is a Jacobian factor between particle rapidity $y$ and pseudorapidity $\eta$ [33].

Equations (6) and (7) establish a one-to-one correspondence between the initial-state energy per unit rapidity $d E_{0} / d \eta_{s} \approx A_{\perp}(e \tau)_{0}$ and the charged particle multiplicity $d N_{\mathrm{ch}} / d \eta$. One crucial feature of this result is that it accounts for the entropy production during the preequilibrium phase, which gives rise to a nontrivial dependence on the initial-state energy density $(e \tau)_{0}^{2 / 3}$ as well as on the transport coefficient $(\eta / s)^{1 / 3}$. Our estimate includes all relevant prefactors, in particular, the constant $C_{\infty}$, which is the property of the hydrodynamic attractor, Eq. (3), and depends on the microscopic physics of equilibration. However, it is striking to observe that, for the different theories shown in Fig. 1, the variation of $C_{\infty}$ is only at $\sim 10 \%$ level. We emphasize that Eq. (6) is entirely based on the macroscopic evolution described by a generic hydrodynamic attractor, which sets it apart from previous parametric estimates of entropy production based on particular microscopic scenarios [34-37].

Centrality dependence of particle multiplicity.-One important phenomenological consequence of the entropy production in the preequilibrium phase concerns the determination of initial conditions for hydrodynamic simulations of heavy ion collisions (see, e.g., [38]). While, strictly speaking, our estimate of the entropy density in Eq. (6) was derived assuming a one dimensional expansion, the influence of transverse gradients can be neglected over the short preequilibrium times, and we can directly promote Eq. (6) to an estimate for the local entropy density, $\left.\tau s\left(\tau, \mathbf{x}_{\perp}\right)\right|_{\tau=\tau_{\text {hydro }}}$. Specifically, the preflow $v_{\perp} \sim$ $\tau_{\text {hydro }} \nabla_{\perp} e / e$ is negligible as long as gradients $\nabla_{\perp} e / e$ are small on the scale of hydrodynamization time $\left(c \tau_{\text {hydro }}\right)$ $[25,26]$, and the one dimensional constitutive relation for $P_{L} / e$ approximately remains valid, as explicitly confirmed in [39]. Effectively, Eq. (6) then provides a nonlinear map of the initial-state energy density profile to the entropy density profile at later times $\tau \sim \tau_{\text {hydro }}$.

Now, in order to illustrate the impact of the preequilibrium phase, we will study the effects on the centrality dependence of the charged particle multiplicity within a simple initial state model based on the color-glass condensate effective theory of high-energy QCD [40]. Within the dilute-dense formulation of this theory [41-45], the initial energy density per unit rapidity $(e \tau)_{0}$ as well as the initial gluon multiplicity per unit rapidity $(n \tau)_{0}$ can be calculated from $\mathbf{k}_{\perp}$ factorization and are given in terms of convolutions of unintegrated gluon distributions [46]. Essentially, one finds that (up to logarithmic corrections) $(e \tau)_{0}$ and $(n \tau)_{0}$ are proportional to the (local) saturation scales $Q_{s}\left(\mathbf{x}_{\perp}\right)$ of the two colliding nuclei $[41,42]$

$$
\begin{gathered}
(e \tau)_{0}\left(\mathbf{x}_{\perp}\right) \propto\left(Q_{s}^{<}\right)^{2}\left(\mathbf{x}_{\perp}\right) Q_{s}^{>}\left(\mathbf{x}_{\perp}\right), \\
(n \tau)_{0}\left(\mathbf{x}_{\perp}\right) \propto\left(Q_{s}^{<}\right)^{2}\left(\mathbf{x}_{\perp}\right),
\end{gathered}
$$

where $Q_{s}^{>/<}$is the saturation scale of the nucleus representing larger or smaller $Q_{s}$ at position $\mathbf{x}_{\perp}$.

Since the saturation scale locally characterizes the longitudinally integrated density of color charge inside the nucleus, it is generically proportional to the nuclear thickness

$$
Q_{s}^{2}\left(\mathbf{x}_{\perp}\right) \propto T\left(\mathbf{x}_{\perp}\right),
$$

whose definition is recalled in the Supplemental Material [47]. Based on these considerations, one can then try to estimate the charged particle multiplicity per unit rapidity from the initial gluon multiplicity $(n \tau)_{0}$ (i.e., with or without preequilibrium) according to

$$
\frac{d N_{\mathrm{ch}}}{d \eta} \propto \int d^{2} \mathbf{x}_{\perp} T^{<}\left(\mathbf{x}_{\perp}\right),
$$

as was done, for example, in [50,51]. However, such an estimate is appropriate only when there is no significant amount of particle production in the final state. Conversely, if the initial state evolves into an almost ideal QGP fluid, one needs to account for the entropy production during the preequilibrium phase. By employing Eqs. (6) and (7) the charged particle multiplicity is then estimated from the initial state energy density $(e \tau)_{0}$, (i.e., with preequilibrium) as

$$
\frac{d N_{\mathrm{ch}}}{d \eta} \propto \int d^{2} \mathbf{x}_{\perp}\left(T^{<}\left(\mathbf{x}_{\perp}\right) \sqrt{T^{>}\left(\mathbf{x}_{\perp}\right)}\right)^{2 / 3} .
$$

We illustrate the difference between the two estimates in the upper panel of Fig. 2, where we compare the centrality dependence of the multiplicity $d N_{\mathrm{ch}} / d \eta$ from Eq. (12) (solid line) and Eq. (11) (dashed line), with the nuclear thickness and centrality quantiles determined from the optical Glauber model (see the Supplemental Material for details [47]). Both estimates are normalized to reproduce the experimentally measured value of $d N_{\mathrm{ch}} / d \eta$ in the $10 \%-20 \%$ centrality class of $\mathrm{Pb}-\mathrm{Pb}$ collisions at $\sqrt{s_{\mathrm{NN}}}=2.76 \mathrm{TeV}$ (center-of-mass energy per nucleon pair). Different trends in the centrality dependence of $d N_{\mathrm{ch}} / d \eta$ are clearly visible, indicating the 


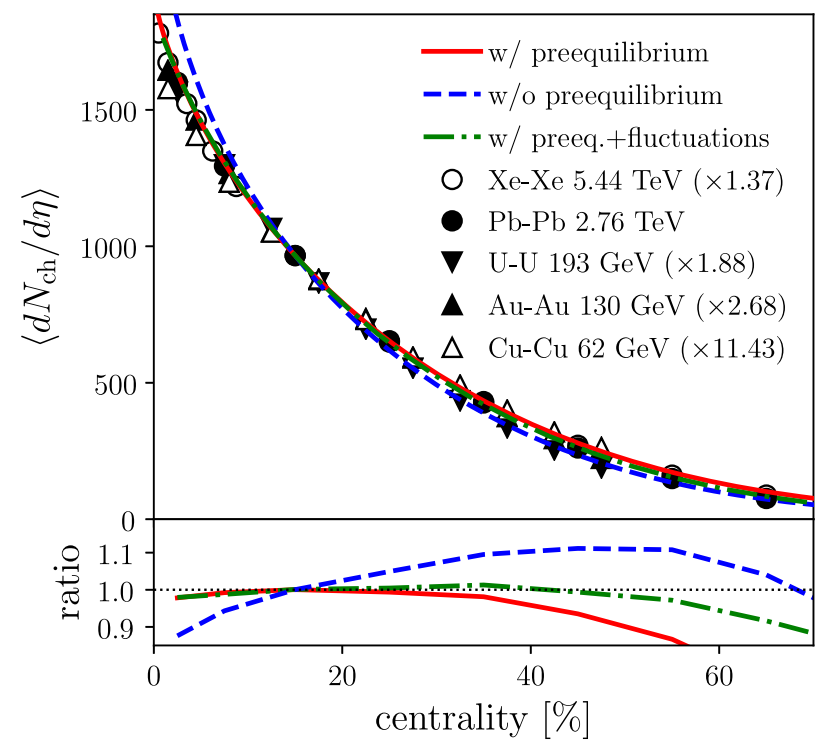

FIG. 2. The effect of preequilibrium dynamics on the centrality dependence of the charged-particle multiplicity, $d N_{\mathrm{ch}} / d \eta$, can be appreciated by comparing the theoretical estimate on the gluon number (blue dashed line), given by Eq. (11), to that on the charged-particle multiplicity after equilibration (red solid line), given by Eq. (12). The green dotted-dashed line includes, as well, fluctuations in the initial state energy within a Glauber Monte Carlo approach. Experimental data points are shown for different collision systems: $\mathrm{Xe}-\mathrm{Xe}$ [52], $\mathrm{Pb}-\mathrm{Pb}$ [53], U-U [54], $\mathrm{Au}-\mathrm{Au}$ [55], and $\mathrm{Cu}-\mathrm{Cu}$ [55] collisions. All curves are normalized to present the same value of multiplicity as ALICE $\mathrm{Pb}-\mathrm{Pb}$ data in the $10 \%-20 \%$ centrality bin. The bottom panel shows the ratio between $2.76 \mathrm{TeV} \mathrm{Pb}-\mathrm{Pb}$ data and the theory curves.

importance of the preequilibrium phase when comparing observables of this type to experimental data.

Since $d N_{\mathrm{ch}} / d \eta$ is accurately measured in experiment for a wide variety of colliding systems and energies, we can also compare the two estimates directly to experimental data, which are reported as symbols in the upper panel of Fig. 2. It is interesting to note that the average $\left\langle d N_{\mathrm{ch}} / d \eta\right\rangle$ as a function of centrality possesses a remarkable degree of universality, such that - up to an overall normalization factor for each collision system-data points for different colliding species ( $\mathrm{Au}, \mathrm{Cu}, \mathrm{Pb}, \mathrm{U}, \mathrm{Xe})$ at RHIC and $\mathrm{LHC}$ energies all collapse onto a single curve to high accuracy. Despite the simplicity of our theoretical estimate, we find that the curve including preequilibrium effects provides a rather good description of the experimental data, except for the more peripheral bins, where fluctuations play an important role (see below). Because of the nontrivial geometry dependence in Eq. (12), the calculation including preequilibrium dynamics provides a much better description of the data than the estimate in Eq. (11), which is based solely on the initial state.

Even though Eq. (12) can be clearly justified from theoretical calculations, our description is by no means unique. Other phenomenological models [56-60] successfully reproduce the centrality dependence seen in Fig. 2 by introducing various sources of event-by-event fluctuations such as number and positions of participant nucleons, their interaction strength, etc. However, it is important to emphasize in this context that the preequilibrium phase also modifies the statistics of fluctuations, such that, for the long wavelength perturbations $[25,26]$,

$$
\frac{\delta s_{\text {hydro }}}{s_{\text {hydro }}}=\frac{2}{3} \frac{\delta e_{0}}{e_{0}},
$$

which follows from the linearization of Eq. (6). While Eq. (12) overpredicts particle production in peripheral collisions, it is, therefore, not surprising that one can restore agreement with peripheral data by including event-by-event fluctuations. This is demonstrated by the dotted-dashed line in Fig. 2, where the average of the nuclear thickness in Eq. (12) has been determined from a Glauber Monte Carlo model [61] (see the Supplemental Material for details [47]).

Estimating the initial-state energy density.-So far, we illustrated the utility of Eq. (6) for describing entropy production in the preequilibrium phase of high-energy heavy-ion collisions. However, an equally important application concerns the inverse problem, namely, the estimation of the energy density $e_{0}$ of the nonequilibrium state at very early time $\tau_{0}$ from experimental measurements of hadrons in the final state.

By inverting Eqs. (6) and (7) (and inserting typical values of $d N_{\mathrm{ch}} / d \eta \sim 1600$ and $A_{\perp} \approx \pi R_{\mathrm{Pb}}^{2} \approx 138 \mathrm{fm}^{2}$ ), we obtain the following estimate for the initial energy density for central $\mathrm{Pb}-\mathrm{Pb}$ collisions at $\sqrt{s_{\mathrm{NN}}}=2.76 \mathrm{TeV}$ :

$$
\begin{aligned}
e_{0} \approx & 270 \mathrm{GeV} / \mathrm{fm}^{3}\left(\frac{\tau_{0}}{0.1 \mathrm{fm} / c}\right)^{-1}\left(\frac{C_{\infty}}{0.87}\right)^{-9 / 8}\left(\frac{\eta / s}{2 / 4 \pi}\right)^{-1 / 2} \\
& \times\left(\frac{A_{\perp}}{138 \mathrm{fm}^{2}}\right)^{-3 / 2}\left(\frac{d N_{\mathrm{ch}} / d \eta}{1600}\right)^{3 / 2} \\
& \times\left(\frac{\nu_{\mathrm{eff}}}{40}\right)^{-1 / 2}\left(\frac{S / N_{\mathrm{ch}}}{7.5}\right)^{3 / 2}
\end{aligned}
$$

at a time $\tau_{0}=0.1 \mathrm{fm} / c$, which should be at least of the order of the formation time $1 / Q_{s} \approx 0.1 \mathrm{fm} / c$ [4], but small compared to the equilibration time $\tau_{\text {hydro }} \approx 1 \mathrm{fm} / c[26,27]$ for the estimate in Eq. (14) to be valid. One finds that the initial energy density quoted in Eq. (14) is nearly 3 orders of magnitude higher than the energy density at the QCD crossover $e_{c} \approx 0.346(41) \mathrm{GeV} / \mathrm{fm}^{3}$ (for $2+1$ flavor QCD) $[62,63]$.

We emphasize that, unlike the usual Bjorken estimate based on the measured final-state energy $e_{0}=$ $\left(1 / \tau_{0} A_{\perp}\right) d E_{\text {final }} / d y$ [12], our estimate in Eq. (14) includes the work done during the expansion of the QGP [11]. We demonstrate this effect in Fig. 3 where we compare the experimentally measured $d E_{\text {final }} / d y$ in the final state to the 


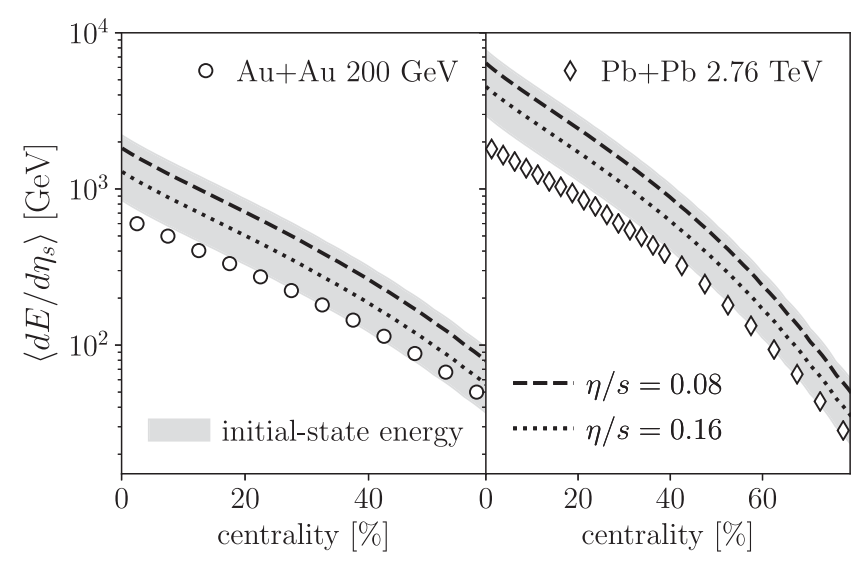

FIG. 3. Estimate of the initial energy per unit space time rapidity $d E_{0} / d \eta_{s}$ (shaded area) determined from the measured particle multiplicity in $\sqrt{s_{\mathrm{NN}}}=200 \mathrm{GeV} \mathrm{Au}-\mathrm{Au}$ (left) and $\sqrt{s_{\mathrm{NN}}}=2.76 \mathrm{TeV} \mathrm{Pb}-\mathrm{Pb}$ collisions (right). Bands correspond to variations of $\eta / s=0.08-0.24$ and $C_{\infty}=0.80-1.15$ while keeping all other parameters fixed as in Eq. (14), and similarly, the dashed lines correspond to specific values of $\eta / s=0.08$, 0.16 . For comparison, we show data points for the experimentally measured final state energy $d E_{\text {final }} / d y$ [33,54], which is smaller due to the work performed against the longitudinal expansion.

initial-state energy per unit rapidity $d E_{0} / d \eta_{s}=\int d \mathbf{x}_{\perp}(e \tau)_{0}$ reconstructed from the measured particle multiplicities as in Eq. (14). Note that, to better account for the nontrivial transverse geometry, we have estimated the transverse area $A_{\perp}$ from our fluctuating initial state model as described in the Supplemental Material [47].

Based on this analysis, we find that, especially in central collisions at high energies, the initial state $d E_{0} / d \eta_{s}$ can easily exceed the measured $d E_{\text {final }} / d y$ in the final state by a factor of 2 to 3 . Evidently, the exact amount of work done during the expansion is subject to uncertainties in the nonequilibrium and transport properties of the QGP, which we quantify by uncertainty bands in Fig. 3, corresponding to variations of $\eta / s$ and $C_{\infty}$ within the anticipated margins $\left(\eta / s=0.08-0.24\right.$ and $\left.C_{\infty}=0.80-1.15\right)$. Vice versa, the size of the uncertainty bands in Fig. 3 also demonstrates the fact that, if the initial state energy density can be determined precisely, e.g., from theoretical calculations, then the experimentally measured $d N_{\mathrm{ch}} / d \eta$ will impose strong constraints on the nonequilibrium evolution of the QGP characterized by $C_{\infty}$ and $\eta / s$. Since, the $d E_{0} / d \eta_{s}$ should always be larger than $d E_{\text {final }} / d y$ due to the work performed against the longitudinal expansion, one can further rule out values in the parameter space, where the initial $d E_{0} / d \eta_{s}$ falls below the experimental data points. Specifically, large values of $\eta / s$ and $C_{\infty}$ can already be ruled out, because the estimated $d E_{0} / d \eta_{s}$ in peripheral collisions turns out to be unphysically small, i.e., below the experimental points of $d E_{\text {final }} / d y$, which provide a lower bound on the initial state energy.

Discussion.-Entropy production in high-energy heavyion collisions occurs predominantly during the earliest stages, when the system is significantly out of equilibrium; therefore, measurements of the charged particle multiplicities-reflecting the total amount of entropy produced in the collision - provide a highly sensitive probe of the preequilibrium dynamics. Based on the concept of hydrodynamic attractors, which give a macroscopic description of the early time dynamics of the QGP, we established, for the first time, a direct relation between the initial-state energy and the final-state entropy. This relation, Eq. (6), is remarkably insensitive to the microscopic details of the approach to equilibrium (see Fig. 1).

By combining the information from $d N_{\mathrm{ch}} / d \eta$ on entropy production and $d E_{\text {final }} / d y$ on the work performed against the longitudinal expansion, we demonstrate that a precise calculation of the initial state energy can impose stringent constraints on the shear viscosity to entropy density ratio $\eta / s$. Based on our extraction of $d E_{0} / d \eta_{s}$, which assumes a scenario of (nearly) complete equilibration, we obtain an upper limit for $\eta / s \lesssim 0.4$ for the most favorable choice of all other parameters. Conversely, for $\eta / s \gtrsim 0.4$, we can not expect the QGP to equilibrate in peripheral nucleus-nucleus collisions (see, also, [30,64]) and our estimates need to be revised. Nontrivial modifications due to incomplete equilibration will arise in this context, which should be investigated further, for instance by means of the KØмРøST preequilibrium package $[26,27,65]$. We expect such effects to become particularly important in collisions of smaller nuclei, e.g., $p$-A or O-O, which may, therefore, provide even deeper insights into the fascinating out-of-equilibrium dynamics of the QGP.

We thank A. Andronic, J. Berges, N. Borghini, M. Heller, C. Klein-Bösing, A. Kurkela, T. Lappi, M. Martinez, J. Y. Ollitrault, K. Reygers, C. Schmidt, D. Teaney, and R. Venugopalan for stimulating discussions and P. Romatschke for also providing the AdS/CFT attractor curve. This work was supported in part by the German Research Foundation (DFG) through the Collaborative Research Centres CRC-TR 211: Strong-interaction matter under extreme conditions Project No. 315477589 (S. S.) and SFB 1225: Isolated quantum systems and universality in extreme conditions (ISOQUANT) (A. M.). We thank the Institute for Nuclear Theory at the University of Washington for its kind hospitality and stimulating research environment.

*giuliano.giacalone@ipht.fr †aleksas.mazeliauskas@cern.ch ‘sschlichting@physik.uni-bielefeld.de

[1] W. Busza, K. Rajagopal, and W. van der Schee, Annu. Rev. Nucl. Part. Sci. 68, 339 (2018).

[2] T. Schäfer and D. Teaney, Rep. Prog. Phys. 72, 126001 (2009).

[3] A. Adams, L. D. Carr, T. Schäfer, P. Steinberg, and J. E. Thomas, New J. Phys. 14, 115009 (2012). 
[4] S. Schlichting and D. Teaney, Annu. Rev. Nucl. Part. Sci. 69, 447 (2019).

[5] J. Adams et al. (STAR Collaboration), Nucl. Phys. A757, 102 (2005).

[6] K. Adcox et al. (PHENIX Collaboration), Nucl. Phys. A757, 184 (2005).

[7] B. B. Back et al., Nucl. Phys. A757, 28 (2005).

[8] I. Arsene et al. (BRAHMS Collaboration), Nucl. Phys. A757, 1 (2005).

[9] W. Florkowski, M. P. Heller, and M. Spalinski, Rep. Prog. Phys. 81, 046001 (2018).

[10] P. Romatschke and U. Romatschke, Relativistic Fluid Dynamics In and Out of Equilibrium, Cambridge Monographs on Mathematical Physics (Cambridge University Press, Cambridge, England, 2019).

[11] M. Gyulassy and T. Matsui, Phys. Rev. D 29, 419 (1984).

[12] J. D. Bjorken, Phys. Rev. D 27, 140 (1983).

[13] M. P. Heller, R. A. Janik, and P. Witaszczyk, Phys. Rev. Lett. 108, 201602 (2012).

[14] M. P. Heller and M. Spalinski, Phys. Rev. Lett. 115, 072501 (2015).

[15] P. Romatschke, Phys. Rev. Lett. 120, 012301 (2018).

[16] M. P. Heller, A. Kurkela, M. Spaliński, and V. Svensson, Phys. Rev. D 97, 091503(R) (2018).

[17] M. Strickland, J. Noronha, and G. S. Denicol, Phys. Rev. D 97, 036020 (2018).

[18] J.-P. Blaizot and L. Yan, Phys. Lett. B 780, 283 (2018).

[19] M. Strickland, J. High Energy Phys. 12 (2018) 128.

[20] A. Behtash, S. Kamata, M. Martinez, and H. Shi, Phys. Rev. D 99, 116012 (2019).

[21] A. Behtash, C. N. Cruz-Camacho, and M. Martinez, Phys. Rev. D 97, 044041 (2018).

[22] A. Behtash, C. N. Cruz-Camacho, S. Kamata, and M. Martinez, Phys. Lett. B 797, 134914 (2019).

[23] A. Kurkela and Y. Zhu, Phys. Rev. Lett. 115, 182301 (2015).

[24] L. Keegan, A. Kurkela, P. Romatschke, W. van der Schee, and Y. Zhu, J. High Energy Phys. 04 (2016) 031.

[25] L. Keegan, A. Kurkela, A. Mazeliauskas, and D. Teaney, J. High Energy Phys. 08 (2016) 171.

[26] A. Kurkela, A. Mazeliauskas, J.-F. Paquet, S. Schlichting, and D. Teaney, Phys. Rev. C 99, 034910 (2019).

[27] A. Kurkela, A. Mazeliauskas, J.-F. Paquet, S. Schlichting, and D. Teaney, Phys. Rev. Lett. 122, 122302 (2019).

[28] A. Kurkela and A. Mazeliauskas, Phys. Rev. Lett. 122, 142301 (2019).

[29] A. Kurkela and A. Mazeliauskas, Phys. Rev. D 99, 054018 (2019).

[30] A. Kurkela, U. A. Wiedemann, and B. Wu, Eur. Phys. J. C 79, 965 (2019).

[31] D. A. Teaney, in Quark-Gluon Plasma 4, edited by R. C. Hwa and X.-N. Wang (World Scientific, Singapore, 2010), pp. 207-266.

[32] P. Hanus, K. Reygers, and A. Mazeliauskas, Phys. Rev. C 100, 064903 (2019).

[33] J. Adam et al. (ALICE Collaboration), Phys. Rev. C 94, 034903 (2016).

[34] R. Baier, A. H. Mueller, D. Schiff, and D. T. Son, Phys. Lett. B 539, 46 (2002).

[35] R. Baier, A. H. Mueller, D. Schiff, and D. T. Son, arXiv: 1103.1259.
[36] W. van der Schee and B. Schenke, Phys. Rev. C 92, 064907 (2015).

[37] J. Berges, K. Reygers, N. Tanji, and R. Venugopalan, Phys. Rev. C 95, 054904 (2017).

[38] U. Heinz and R. Snellings, Annu. Rev. Nucl. Part. Sci. 63, 123 (2013).

[39] A. Kurkela, W. van der Schee, U. A. Wiedemann, and B. Wu, arXiv:1907.08101.

[40] F. Gelis, E. Iancu, J. Jalilian-Marian, and R. Venugopalan, Annu. Rev. Nucl. Part. Sci. 60, 463 (2010).

[41] A. Dumitru and L. D. McLerran, Nucl. Phys. A700, 492 (2002).

[42] T. Lappi, Phys. Lett. B 643, 11 (2006).

[43] J. P. Blaizot, F. Gelis, and R. Venugopalan, Nucl. Phys. A743, 13 (2004).

[44] J.-P. Blaizot and Y. Mehtar-Tani, Nucl. Phys. A818, 97 (2009).

[45] J. P. Blaizot, T. Lappi, and Y. Mehtar-Tani, Nucl. Phys. A846, 63 (2010).

[46] We note that explicit comparisons of dilute-dense and dense-dense calculations in Ref. [45] show that, in the momentum range $p_{T} \gtrsim Q_{s}$, which gives the dominant contribution to $d E / d y$, the dilute-dense $k_{T}$ factorization calculations accurately describe dense-dense classical YangMills results.

[47] See Supplemental Material at http://link.aps.org/ supplemental/10.1103/PhysRevLett.123.262301 which provides the description of the Monte Carlo Glauber model of nucleus-nucleus collisions used to determine the nuclear thickness functions, which are needed for the calculation of the initial-state energy profile. In addition, we provide detailed formulas for calculating the centrality dependence of the averaged charged-particle multiplicity and the initialstate energy per rapidity, i.e., the lines and shaded bands in Figs. 2 and 3 of the manuscript. The supplemental material also includes Refs. [48,49].

[48] H. De Vries, C. W. De Jager, and C. De Vries, At. Data Nucl. Data Tables 36, 495 (1987).

[49] J. L. Albacete and C. Marquet, Prog. Part. Nucl. Phys. 76, 1 (2014).

[50] M. Mace, V. V. Skokov, P. Tribedy, and R. Venugopalan, Phys. Rev. Lett. 121, 052301 (2018); 123, 039901(E) (2019).

[51] B. Schenke, P. Tribedy, and R. Venugopalan, Phys. Rev. C 86, 034908 (2012).

[52] S. Acharya et al. (ALICE Collaboration), Phys. Lett. B 790, 35 (2019).

[53] K. Aamodt et al. (ALICE Collaboration), Phys. Rev. Lett. 106, 032301 (2011).

[54] A. Adare et al. (PHENIX Collaboration), Phys. Rev. C 93, 024901 (2016).

[55] B. Alver et al. (PHOBOS Collaboration), Phys. Rev. C 83, 024913 (2011).

[56] H. J. Drescher and Y. Nara, Phys. Rev. C 75, 034905 (2007).

[57] B. Alver, M. Baker, C. Loizides, and P. Steinberg, arXiv: 0805.4411.

[58] C. Gale, S. Jeon, B. Schenke, P. Tribedy, and R. Venugopalan, Phys. Rev. Lett. 110, 012302 (2013).

[59] H. Niemi, K. J. Eskola, and R. Paatelainen, Phys. Rev. C 93, 024907 (2016). 
[60] J. E. Bernhard, J. S. Moreland, S. A. Bass, J. Liu, and U. Heinz, Phys. Rev. C 94, 024907 (2016).

[61] M. L. Miller, K. Reygers, S. J. Sanders, and P. Steinberg, Annu. Rev. Nucl. Part. Sci. 57, 205 (2007).

[62] H.-T. Ding, F. Karsch, and S. Mukherjee, Int. J. Mod. Phys. E 24, 1530007 (2015).

[63] A. Bazavov et al. (HotQCD Collaboration), Phys. Lett. B 795, 15 (2019).
[64] R. S. Bhalerao, J.-P. Blaizot, N. Borghini, and J.-Y. Ollitrault, Phys. Lett. B 627, 49 (2005).

[65] A. Kurkela, A. Mazeliauskas, J.-F. Paquet, S. Schlichting, and D. Teaney, KøMPøST: Linearized kinetic theory propagator of initial conditions for heavy ion collisions, https:// github.com/KMPST/KoMPoST (2018). 